\title{
Quantum Zeno Effect for Exponentially Decaying Systems
}

\author{
Kazuki Koshind* \\ Core Research for Evolutional Science and Technology (CREST), \\ Japan Science and Technology Corporation (JST), \\ c/o Department of Physical Science, Graduate School of Engineering Science, \\ Osaka University, Toyonaka, Osaka 560-8531, Japan \\ Akira Shimizu丹 \\ Department of Basic Science, University of Tokyo, 3-8-1 Komaba, Tokyo 153-8902, Japan
}

(Dated: November 19, 2018)

\begin{abstract}
The quantum Zeno effect - suppression of decay by frequent measurements - was believed to occur only when the response of the detector is so quick that the initial tiny deviation from the exponential decay law is detectable. However, we show that it can occur even for exactly exponentially decaying systems, for which this condition is never satisfied, by considering a realistic case where the detector has a finite energy band of detection. The conventional theories correspond to the limit of an infinite bandwidth. This implies that the Zeno effect occurs more widely than expected so far.
\end{abstract}

PACS numbers: 03.65.Xp,06.20.Dk,03.65.Yz

The survival probability $s(t)$ of an unstable quantum system generally decreases quadratically with time $t$ at the beginning of the decay, and later follows the wellknown exponentially decay law. The crossover takes place at $t \sim \tau_{j}$, which is called the jump time. Noticing this fact and simply applying the projection postulate on measurements, it was predicted that frequent measurements with time intervals $\tau$ result in the suppression of the decay if $\tau$ is small enough to satisfy $\tau \lesssim \tau_{j}[1]$. Similarly, the suppression was supposed to occur by continuous measurement using an apparatus with short response time $\tau$ satisfying $\tau \lesssim \tau_{j}[2]$. This prediction, called the quantum Zeno effect (QZE), is one of the most curious results of the quantum measurement, and has been attracting much attention both theoretically $1,2,2,3,4,5,6,7]$ and experimentally $[8,9,10,11,12]$. However, in most unstable systems, $\tau_{j}$ is extremely small so that the condition $\tau \lesssim \tau_{j}$ cannot be satisfied by existing detectors. It has been therefore believed that the QZE would not occur in most unstable systems.

However, these conventional arguments on the QZE need to be reconsidered, because, in general, real measurement processes are not 'projective measurements' that are described by the projection postulate 13, 14, 15. A more general and accurate way of analyzing measurement processes is to apply the laws of quantum theory to a larger system which include both the original quantum system to be measured and a part of measuring apparatus 14, 15. Such an approach has been successfully applied, e.g., to quantum optics [15, 16, 17, 18] and the QZE $[2,7]$. This enables one to study not only the temporal evolution of the unstable system but also the response of the measuring apparatus. Moreover, one can discuss gen-

\footnotetext{
*Electronic address: ikuzak@aria.mp.es.osaka-u.ac.jp

${ }^{\dagger}$ Electronic address: shmz@ASone.c.u-tokyo.ac.jp
}

eral measurement processes such as measurement with a finite probability of error 7, 18.

In this paper, we apply this modern approach to analyze the QZE in a realistic situation where the unstable system is monitored continuously using a measuring apparatus (detector) with a finite energy band of detection. Furthermore, we consider the case of 'indirect measurement,' in which the detector acts only on the decay products, because some of the previous works assuming direct interactions have often been criticized as not being a genuine QZE. We show that the QZE can take place even in systems that exactly follow the exponential decay law, for which $\tau_{j}$ is infinitesimal hence the conventional condition for the QZE, $\tau \lesssim \tau_{j}$, is never satisfied. We clarify its physical origin, derive the conditions for inducing the QZE in such systems, and show that the conventional projection-based theory can be reproduced in the ideal, but unrealistic limit of an infinite detection bandwidth. Our results imply that the response time required for the QZE is not so short, the required jump time is not so long, and hence the QZE may take place much more widely than expected so far.

As the unstable quantum system we consider the excited state of a two-level atom, which decays to the ground state with a finite lifetime accompanying emission of a photon. By detecting the emitted photon, the observer can know the decay of the atom. As the photon detection process, we consider a standard one 15, 16, 17]: In the photodetector, the emitted photon is absorbed by a semiconductor and an electron-hole pair is generated. It finally yields a macroscopic signal after magnification processes, by which the observer knows the decay of the excited atom. Following the quantum theory of photon counting 15, 16, 17, we treat the relevant part of the detector, i.e., electron-hole pairs, as a part of the total quantum system. The electron-hole pairs can be described as bosonic elementary excitations, because their density is low in the detection process. The total quantum system 
is therefore composed of three parts: (i) A two-level atom with the ground state $|g\rangle$ and the excited state $|x\rangle$ with the transition energy $\Omega$ (we hereafter take $\hbar=c=1$ ), (ii) the photon field, whose eigenmodes are labeled by the wavevector $\boldsymbol{k}$, and (iii) the field of the electron-hole bosonic excitation in the detector. By taking the energy of $|g\rangle$ as the origin of the energy, the Hamiltonian of the total system is given as follows:

$$
\begin{aligned}
\mathcal{H} & =\mathcal{H}_{0}+\mathcal{H}_{1}+\mathcal{H}_{2}, \\
\mathcal{H}_{0} & =\Omega|\mathrm{x}\rangle\langle\mathrm{x}| \\
\mathcal{H}_{1} & =\int \mathrm{d} \boldsymbol{k}\left[\left(\xi_{\boldsymbol{k}}|x\rangle\langle g| b_{\boldsymbol{k}}+\text { H.c. }\right)+k b_{\boldsymbol{k}}^{\dagger} b_{\boldsymbol{k}}\right], \\
\mathcal{H}_{2} & =\iint \mathrm{d} \boldsymbol{k} \mathrm{d} \omega\left[\left(\sqrt{\eta_{\boldsymbol{k}}} b_{\boldsymbol{k}}^{\dagger} c_{\boldsymbol{k} \omega}+\text { H.c. }\right)+\omega c_{\boldsymbol{k} \omega}^{\dagger} c_{\boldsymbol{k} \omega}\right]
\end{aligned}
$$

Here, $\mathcal{H}_{1}$ represents the atom-photon interaction, where $b_{\boldsymbol{k}}$ is the annihilation operator for the photon with wavevector $\boldsymbol{k}$, whose energy is $k=|\boldsymbol{k}|$. The atom-photon part $\mathcal{H}_{0}+\mathcal{H}_{1}$ constitutes the original unstable quantum system to be measured. $\mathcal{H}_{2}$ represents the interaction between the emitted photon and the detector. Every photon mode is coupled to a continuum of the bosonic elementary excitations in the detector, whose annihilation operator is denoted by $c_{\boldsymbol{k} \omega}$. The commutation relations are orthonormalized as $\left[b_{\boldsymbol{k}}, b_{\boldsymbol{k}^{\prime}}^{\dagger}\right]=\delta\left(\boldsymbol{k}-\boldsymbol{k}^{\prime}\right)$ and $\left[c_{\boldsymbol{k} \omega}, c_{\boldsymbol{k}^{\prime} \omega^{\prime}}^{\dagger}\right]=\delta\left(\boldsymbol{k}-\boldsymbol{k}^{\prime}\right) \delta\left(\omega-\omega^{\prime}\right)$.

In order to demonstrate that the QZE does occur even when $\tau \gtrsim \tau_{j}$, for which the QZE cannot be expected according to the conventional theories, we study the limiting case of infinitesimal $\tau_{j}$ [19]. Namely, we investigate the case where the survival probability $s(t)$ of the initial excited state exactly follows the exponential decay law, $s(t)=\exp (-\gamma t)$, when the original system is not measured, i.e., when $\mathcal{H}_{2}=0$. In the present model, we obtain such an exact exponential decay for the original quantum system (described by $\mathcal{H}_{0}+\mathcal{H}_{1}$ ) by putting the atom-photon coupling after angular integration to be independent of the photon energy $k$;

$$
\int \mathrm{d} \boldsymbol{q}\left|\xi_{\boldsymbol{q}}\right|^{2} \delta(|\boldsymbol{q}|-k) \equiv \frac{\gamma}{2 \pi},
$$

and by extending the lower limit of the photon energy to $-\infty$. In this case, $\tau_{j}$ becomes infinitesimal, and $\tau>\tau_{j}$ for any finite $\tau$. We show in the following that the QZE can occur even in this case [19]. The point is that any real detector has a finite bandwidth of detection. In contrast, the use of the projection hypothesis corresponds to the use of an ideal but unrealistic detector that has an infinite bandwidth.

To demonstrate this, we here take the following simple form for $\eta_{k}$;

$$
\eta_{k}=\eta_{k} \equiv \frac{\eta / 2 \pi}{1+[(k-\Omega) / \Delta]^{n}}
$$

where $n$ is a large even integer (we take $n=6$ in the numerical examples). In this case, photons within the

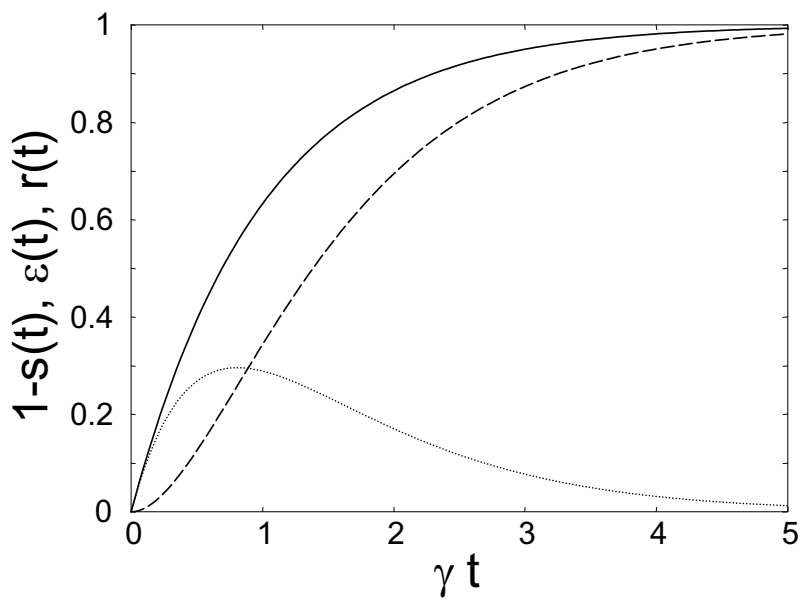

FIG. 1: Temporal evolution of $1-s(t)$ (solid curve), $\epsilon(t)$ (dotted), and $r(t)$ (broken). The parameters are chosen as $2 \pi \Delta / \gamma=100$ and $\eta / \gamma=1.5$. It is seen that $r(t)$ follows $1-s(t)$ with a delay time about $\tau \equiv \eta^{-1}$.

energy range $|k-\Omega| \lesssim \Delta$ are counted by the detector with a timescale of $\eta^{-1} \equiv \tau$, while photons outside of this energy range are not counted. Thus, the detector has a detection band centered around the atomic transition energy $\Omega$ with the bandwidth $\simeq 2 \Delta$. The atomic decay is continuously monitored with this detector.

To see the temporal evolution of the whole system, we put $|\psi(t)\rangle=e^{-\mathrm{i} \mathcal{H} t}|\mathrm{x}, 0,0\rangle=f(t)|\mathrm{x}, 0,0\rangle+$ $\int \mathrm{d} \boldsymbol{k} f_{\boldsymbol{k}}(t)|\mathrm{g}, \boldsymbol{k}, 0\rangle+\iint \mathrm{d} \boldsymbol{k} \mathrm{d} \omega f_{\boldsymbol{k} \omega}(t)|\mathrm{g}, 0, \boldsymbol{k} \omega\rangle$, and define three probabilities of physical interest; $s(t)=|f(t)|^{2}$ (survival probability of the atom), $\varepsilon(t)=\int \mathrm{d} \boldsymbol{k}\left|f_{\boldsymbol{k}}(t)\right|^{2}$ (probability that the atom has decayed but the emitted photon is not absorbed by the detector), and $r(t)=$ $\iint \mathrm{d} \boldsymbol{k} \mathrm{d} \omega\left|f_{\boldsymbol{k} \omega}(t)\right|^{2}$ (probability that the emitted photon is absorbed). $r(t)$ can be interpreted as the probability of getting a detector response, whereas $\varepsilon(t)$ is the probability that the detector reports an erroneous result. One of the advantages of the present theory is that all of these interesting quantities can be calculated. A numerical example is shown in Fig. 1] Since $\Delta \gg \gamma$ in this example, the atomic natural linewidth $(\sim \gamma)$ is completely covered by the detection band $(\sim \Delta)$, and the emitted photon is counted almost perfectly $(r(t) \simeq 1$ for $t \rightarrow \infty)$. It is observed that $r(t)$ follows the decay probability $1-s(t)$ with a delay time $\simeq \tau\left(=\eta^{-1}\right)$. Hence, $\tau$ is the response time of the detector. To be more precise, $\tau$ is the lower limit of the response time because additional delays in the response, such as delays in signal magnification processes, may occur in practical experiments. In discussing fundamental physics, the limiting value is more significant than practical values, which depend strongly on detailed experimental conditions. A typical value of $\tau$ for GaAs is $10^{-15} \mathrm{~s}$, which is much shorter than the practical response times of commercial photodetectors, which range from $10^{-6}$ to $10^{-13} \mathrm{~s}$.

In order to see the QZE, we investigate how the survival probability $s(t)$ is affected by the detector parame- 
ters such as the response time $\tau$ and the detection bandwidth $\Delta$ of the detector. For this purpose, we transform $\mathcal{H}$ into the following renormalized form [],

$$
\begin{aligned}
\mathcal{H} & =\mathcal{H}_{0}+\overline{\mathcal{H}}_{1}+\overline{\mathcal{H}}_{2}, \\
\overline{\mathcal{H}}_{1} & =\int \mathrm{d} \mu\left[\left(g_{\mu}|x\rangle\langle g| B_{k}+\text { H.c. }\right)+\mu B_{\mu}^{\dagger} B_{\mu}\right],
\end{aligned}
$$

where $\overline{\mathcal{H}}_{2}$ is composed of terms which are decoupled from the atom. Here, $B_{\mu}$ is a coupled-mode operator that is a linear combination of $b_{\boldsymbol{k}}$ 's and $c_{\boldsymbol{k} \omega}$ 's 7], which is orthonormalized in terms of a one-dimensional label $\mu$ as $\left[B_{\mu}, B_{\mu^{\prime}}^{\dagger}\right]=\delta\left(\mu-\mu^{\prime}\right)$, and $\left|g_{\mu}\right|^{2}$ is given by

$$
\left|g_{\mu}\right|^{2}=\frac{\gamma}{2 \pi} \int \mathrm{d} k \frac{\eta_{k}}{\left|\mu-k-\mathrm{i} \pi \eta_{k}\right|^{2}},
$$

which we call the renormalized form factor. Thus, the atom is coupled to a single continuum of $B_{\mu}$, with the renormalized form factor. When the atom-photon system is free from the measurement, i.e., when $\eta=0$, the form factor reduces to the 'free' value, $\left|g_{\mu}\right|^{2}=\gamma / 2 \pi$, and the atomic decay exactly follows the exponential law, $s(t)=\exp (-\gamma t)$. When the detector is present, on the other hand, $\left|g_{\mu}\right|^{2}$ is modified (renormalized) as shown in Fig. 2] where $\left|g_{\mu}\right|^{2}$ is plotted for several values of $\eta / \Delta$. It is seen that $\left|g_{\mu}\right|^{2}$ is decreased for $|\mu-\Omega| \lesssim \Delta$ (inside the detection band) and is increased for $|\mu-\Omega| \gtrsim \Delta$ (outside). For larger $|\mu-\Omega|$, modification of $\left|g_{\mu}\right|^{2}$ becomes small, approaching zero (i.e., $\left|g_{\mu}\right|^{2} \rightarrow \gamma / 2 \pi$ ) for $|\mu-\Omega| \gg \Delta$. These modifications take place satisfying a sum rule, $\int \mathrm{d} \mu\left|g_{\mu}\right|^{2}=$ constant, which is derived from Eq. (91). The figure also shows that the modification of $\left|g_{\mu}\right|^{2}$ occurs more significantly for larger $\eta / \Delta$. This can be seen clearly in the limit of $n \rightarrow \infty$, where $\left|g_{\mu}\right|^{2}$ can be analytically evaluated and its value at the atomic transition energy is given by $\left|g_{\Omega}\right|^{2}=\left(\gamma / \pi^{2}\right) \arctan (2 \Delta / \eta)$. Thus, $\left|g_{\Omega}\right|^{2}$ becomes significantly smaller than the free value $\gamma / 2 \pi$ when $\eta / \Delta \gtrsim 1$, i.e., when

$$
\tau \cdot \Delta \lesssim 1
$$

The survival probability $s(t)$ is governed by the functional form of $\left|g_{\mu}\right|^{2}$. In order to visualize the decay rate, $\ln s(t) /(\gamma t)$ is plotted in Fig. [3] The figure clarifies the following two-stage behavior of the atomic decay: In the first stage $\left(t \lesssim \Delta^{-1}\right)$, the atom decays with the free decay rate $\gamma$, while, in the second stage $\left(t \gtrsim \Delta^{-1}\right)$, the atom decays with a suppressed decay rate $2 \pi\left|g_{\Omega}\right|^{2}$. For the values of parameters chosen in Fig. 3] the atom is kept almost undecayed $(s(t) \simeq 1)$ in the first stage, and significant decay occurs in the second stage with the suppressed decay rate. Namely, the QZE surely takes place, although the system exhibits an exact exponential decay in the absence of measurement.

The two-stage behavior can be understood with a help of the perturbation theory. Applying the lowest-order perturbation to the renormalized form Eq. (7), we obtain

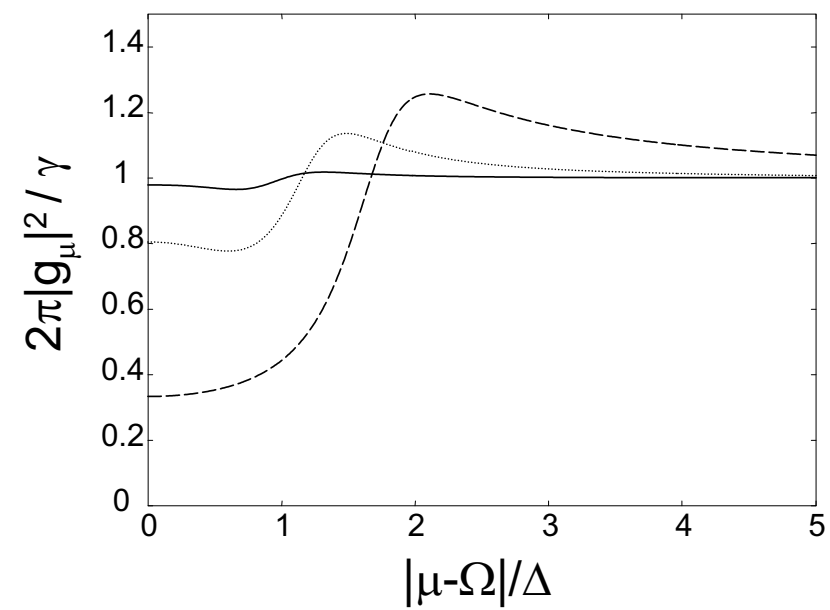

FIG. 2: Plot of $\left|g_{\mu}\right|^{2} \cdot \eta / 2 \pi \Delta$ is chosen at 0.01 (solid curve), 0.1 (dotted), and 1 (broken).

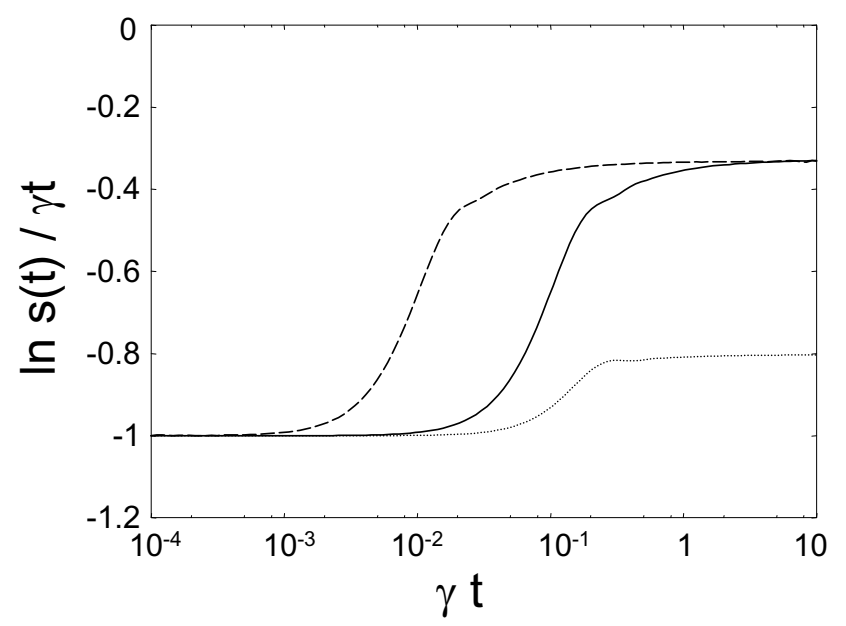

FIG. 3: Plot of $\ln s(t) /(\gamma t)$, i.e., the time dependence of the decay rate. $\{2 \pi \Delta / \gamma, \eta / \gamma\}$ are chosen at $\{100,100\}$ (solid curve), $\{100,10\}$ (dotted), and $\{1000,1000\}$ (broken) The decay rate changes from the free rate $\gamma$ to the suppressed rate $2 \pi\left|g_{\Omega}\right|^{2}$ at $t \sim \Delta^{-1}$.

the decay probability as

$$
1-s(t)=\int \mathrm{d} \mu\left|g_{\mu}\right|^{2} \frac{\sin ^{2}[(\mu-\Omega) t / 2]}{[(\mu-\Omega) / 2]^{2}} .
$$

Taking into account that the main contribution in the integral comes from the region of $\mu$ satisfying $|\mu-\Omega| \lesssim$ $2 \pi t^{-1}$, we evaluate the right-hand side in two cases: In the case of $t \ll \Delta^{-1},\left|g_{\mu}\right|^{2}$ can be approximated by $\left|g_{\infty}\right|^{2}=\gamma / 2 \pi$, which gives the free decay rate $1-s(t)=$ $\gamma t$; whereas in the opposite case of $t \gg \Delta^{-1},\left|g_{\mu}\right|^{2}$ can be approximated by $\left|g_{\Omega}\right|^{2}$, which gives the suppressed decay rate $1-s(t)=2 \pi\left|g_{\Omega}\right|^{2} t$. Thus, the decay rate changes from the free rate to the suppressed rate at $t \sim \Delta^{-1}$.

The QZE occurs for exponentially decaying systems when the following two conditions are satisfied: (i) The transition from the first to the second stage should occur 
before the atom decays. Since the survival probability at $t \sim \Delta^{-1}$ is given by $s\left(\Delta^{-1}\right) \simeq \exp (-\gamma / \Delta)$, this condition is expressed as

$$
\gamma / \Delta \ll 1,
$$

which means that the detection band should completely cover the natural linewidth of the atom. (ii) The decay rate in the second stage $\left(t \gtrsim \Delta^{-1}\right)$ should be significantly suppressed from the free decay rate. This condition is expressed by inequality (10).

The latter condition explains why the QZE was not obtained for exponentially decaying systems by theories based on the projection hypothesis. By applying the projection operator, the quantum coherences between $|x, 0\rangle$ and $|g, \boldsymbol{k}\rangle$ 's are destroyed regardlessly of the energy of the emitted photon. Therefore, the projection-based theory corresponds to $\Delta \rightarrow \infty$ 20. In such a limit, however, inequality (10) cannot be satisfied and the QZE never occurs. Since $\Delta$ of any real detector is finite, such a limit is rather unphysical.

To conclude this paper, we make three remarks. We have presented results for the case where the transition energy $\Omega$ is inside the detection band. It is worth mentioning that if the transition energy $\Omega$ lies outside the detection band, the 'anti QZE' is possible, which is the enhancement of the decay by the measurement [5, [6,, 7$]$. This may be understood from Fig. 22 which shows that $\left|g_{\mu}\right|^{2}>\gamma / 2 \pi$ for $|\mu-\Omega| \gtrsim \Delta$.

We have assumed continuous measurement. Regarding frequent discrete measurements, we note that real detectors have finite response times $\tau$, although the conventional theories on the QZE often assumed that each measurement was an instantaneous projective measurement. Since each measurement takes a detector a time $\tau$, the intervals of the measurements should be longer than $\tau$. Therefore, our case of continuous measurement corresponds to the most frequent discrete measurements using the detector that is described by Eq. (4). In some cases, continuous measurement with the response time $\tau$ corresponds to frequent instantaneous measurements with the intervals of order $\tau$ [2]. However, it should be stressed that this does not mean that continuous measurement always corresponds to some frequent instantaneous projective measurements. In fact, the result of this work is a counterexample.

Finally, we remark that the QZE is closely related to the cavity quantum electrodynamics (QED) 21]. In fact, Eq. (4) is similar to the equation describing the cavity QED where $b_{\boldsymbol{k}}$ 's correspond to discretized cavity modes while $c_{\boldsymbol{k} \omega}$ 's correspond to leaky modes, or, when the cavity is composed of photoabsorptive materials, to excitations in the material. In principle, these quanta can be utilized for detection of the decay, if appropriate magnification (and, if necessary, detection) processes are followed. In such cases, the lower limits of the response time $\tau$ would be determined in terms of the material parameters such as $\eta_{k}$. The present theory not only demonstrates the QZE for the exponentially decaying systems, but also reveals that the cavity QED is related to the QZE through such implicit relations [22].
[1] B. Misra and E. C. G. Sudarshan, J. Math. Phys. 18, 756 (1977).

[2] L. S. Schulman, Phys. Rev. A 57, 1509 (1998).

[3] A. Peres and A. Ron, Phys. Rev. A 42, 5720 (1990).

[4] A. D. Panov, Ann. Phys. 249, 1 (1996).

[5] A. G. Kofman and G. Kurizki, Nature 405, 546 (2000).

[6] P. Facchi, H. Nakazato, and S. Pascazio, Phys. Rev. Lett. 86, 2699 (2001).

[7] K. Koshino and A. Shimizu, Phys. Rev. A 67, 042101 (2003).

[8] W. M. Itano, D. J. Heinzen, J. J. Bollinger, and D. J. Wineland, Phys. Rev. A 41, 2295 (1990).

[9] P. Knight, Nature 344, 493 (1990).

[10] B. Nagels, L. J. F. Hermans, and P. L. Chapovsky, Phys. Rev. Lett. 79, 3097 (1997).

[11] Chr. Balzer, R. Huesmann, W. Neuhauser, and P. E. Toschek, Opt. Commun. 180, 115 (2000).

[12] M. C. Fischer, B. Gutierrez-Medina, and M. G. Raizen, Phys. Rev. Lett. 87, 040402 (2001).

[13] M. A. Nielsen and I. L. Chuang, Quantum Computation and Quantum Information (Cambridge Univ. Press, 2000).
[14] A. Shimizu and K. Fujita, Proc. Quantum Control and Measurement '92, (H. Ezawa and Y. Murayama, eds., Elsevier, 1993), p.191; quant-ph/9804026

[15] Quantum Noise, C. W. Gardiner, P. Zoller, (Springer, Berlin, ed. 2, 2000).

[16] Optical Coherence and Quantum Optics, L. Mandel and E. Wolf, (Cambridge Univ. Press, Cambridge, 1995).

[17] R. J. Glauber, Phys. Rev. 130, 2529 (1963).

[18] A. Shimizu, Phys. Rev. A 43, 3819 (1991).

[19] Although we present the results only for $\tau_{j} \rightarrow 0$, a similar result is obtained for the case of finite $\tau_{j}$.

[20] In fact, for unstable systems with finite $\tau_{j}$, it has been confirmed in Ref. 7] that the present formalism completely reproduces the projection-based theories in the limit of $\Delta \rightarrow \infty$.

[21] Cavity Quantum Electrodynamics, P. R. Berman, Ed., (Academic, San Diego, 1994).

[22] In cavity QED systems, the atomic decay is more sensitive to $\eta_{k}$ than the QZE systems because of the large jump time $\tau_{j}$ due to the discreteness of the cavity mode. 